\title{
Formoterol and beclomethasone versus higher dose beclomethasone as maintenance therapy in adult asthma
}

\author{
D. Bouros*, N. Bachlitzanakis**, J. Kottakis ${ }^{+}$, P. Pfister ${ }^{+}$, V. Polychronopoulos ${ }^{++}$, E. Papadakis*, \\ S. Constantopoulos ${ }^{\star}$, M. Froudarakis*, L. Sichletidis ${ }^{\S}$, N. Siafakas*
}

Formoterol and beclomethasone versus higher dose beclomethasone as maintenance therapy in adult asthma. D. Bouros, N. Bachlitzanakis, J. Kottakis, P. Pfister, V. Polychronopoulos, E. Papadakis, S. Constantopoulos, M. Froudarakis, L. Sichletidis, N. Siafakas. (C) ERS Journals Ltd 1999.

ABSTRACT: A total of 132 adult asthmatics who were symptomatic on $500 \mu \mathrm{g} \cdot \mathrm{day}^{-1}$ inhaled beclomethasone dipropionate (BDP) were studied in an open-label randomized, parallel group, 12 week, clinical trial.

The addition of $12 \mu \mathrm{g}$ formoterol fumarate solution aerosol (pressurized metered dose inhaler) b.i.d. to BDP at a dose of $500 \mu \mathrm{g} \cdot \mathrm{day}^{-1}$ was compared with a higher dose of $1,000 \mu \mathrm{g} \cdot$ day $^{-1} \mathrm{BDP}$.

Mean morning premedication peak expiratory flow rate (PEF) during the final week of treatment (primary end-point) increased in both groups compared to baseline. The estimated treatment difference of $20.4 \mathrm{~L} \cdot \mathrm{min}^{-1}(95 \%$ confidence interval 3.2 37.6) after 12 weeks of treatment was statistically significant $(p<0.05)$ in favour of the formoterol/BDP group. The overall mean morning premedication PEF for the entire treatment period was higher in the formoterol/BDP group $(\mathrm{p}=\mathbf{0 . 0 0 2})$. The overall number of puffs of rescue medication and asthma symptom scores were less in the formoterol/BDP group $(\mathbf{p}<\mathbf{0 . 0 1})$. Safety and tolerability evaluations were satisfactory in both groups.

In conclusion, the results suggest that the addition of formoterol fumarate to the existing dose of an inhaled corticosteroid should be considered as an alternative to increasing the dose of inhaled corticosteroid in the inadequately controlled asthmatic. Eur Respir J 1999; 14: 627-632.
*Depts of Pneumonology and Clinical Pharmacology, Medical School, University of Crete, and University General Hospital, Heraklion, Crete, Greece; **Venizelio Hospital, Crete, Greece; ${ }^{+}$Novartis Pharma AG, Basel Switzerland; ${ }^{++}$A. Fleming Hospital, Athens, "Sotiria Hospital, Athens, "University Hospital, Ioannina, ${ }^{5}$ University Hospital, Thessaloniki, Greece.

Correspondence: D. Bouros, Depts of Pneumonology and Clinical Pharmacology, Medical School, University of Crete, Heraklion, Crete, Greece 71110. Fax: 30 81542650

Keywords: Asthma, beclomethasone, clinical trial, formoterol, long-acting $\beta_{2}$-agonists, treatment

\section{Received: April 41998}

Accepted after revision May 311999

Presented in part at the annual meeting of the European Respiratory Society, Sept 20-24, 1997, Berlin, Germany.Supported by a grant from Novartis, Hellas.
There is a tendency to introduce the option of the regular addition of a long-acting $\beta_{2}$-agonist on existing corticosteroid doses in milder asthmatics earlier than previously proposed. This is also reflected in evolving international guidelines for asthma management [1-2].

This, in the light of the ongoing debate concerning the regular use of $\beta_{2}$-agonists and their potential detrimental effects [3-6], generates the need for experimental studies properly designed to evaluate efficacy and to assess benefit/risk ratio of the suggested regimen.

Limited data are currently available. Previous work by GreENing et al. [7] and Woolcock et al. [8] suggested that the regular addition of salmeterol xinofoate to inhaled beclomethasone dipropionate (BDP) at existing doses ranging $400-1000 \mu \mathrm{g} \cdot \mathrm{day}^{-1}$ resulted in better asthma control than increasing the dose of BDI. Work recently reported on formoterol delivered via a dry powder inhaler (DPI), is also in support of the proposed option [9].

It was therefore hypothesized that adding formoterol to a relatively low dose of an inhaled corticosteroid, in patients still presenting with symptoms, could have similar results to those attained by increased doses of corticosteroids.

To test this hypothesis, a multicentre, randomized, openlabel study was conducted using a population of patients still presenting with symptoms despite a daily dose of 500 $\mu \mathrm{g}$ inhaled BDP. In these patients, the administration of 12 $\mu \mathrm{g}$ formoterol fumarate aerosol solution (from a pressurized metered dose inhaler (pMDI)) given as a combined regimen with $250 \mu \mathrm{g}$ BDP aerosol (pMDI) twice daily, was compared with that of BDP aerosol given at a dose of 500 $\mu \mathrm{g}$ twice daily. This comparison was undertaken over a 12week period.

\section{Methods}

\section{Protocol}

This was a randomized, open-label, between-patient clinical study, which was performed in 11 centres in Greece between July 1995 and November 1996. Ethics Committee approvals for each centre, and Regulatory Authority approval for the protocol and informed consent document were secured as required. All patients provided informed consent prior to initiation of any study procedure. A run-in period of 2 weeks, for patients who were previously on treatment with inhaled BDP aerosol at a constant dose of $500 \mu \mathrm{g}$ daily for at least 1 month, facilitated the establishment of eligibility for subsequent randomization, and served as the baseline for the analyses. Following this runin period, eligible patients were randomized in a blinded fashion to receive either formoterol fumarate aerosol $12 \mu \mathrm{g}$ 
b.i.d. followed by BDP aerosol $250 \mu \mathrm{g}$ b.i.d. or monotherapy with BDP aerosol $500 \mu \mathrm{g}$ b.i.d. for a period of 3 months. During this comparative treatment period, patients returned to the clinic after $4,6,8$ and 12 weeks for assessment and lung function testing.

At the initial screening (visit 1$), \beta_{2}$-agonists and other anti-asthma medication were removed (except BDP). Patients were provided with salbutamol pMDI (Aerolin $\mathbb{R}$ ) $100 \mu \mathrm{g} \cdot$ puff $^{-1}$, GlaxoWellcome, London, UK) to be used for rescue purposes on an "as needed" basis. A spacer device (Optihalerß), Healthscan Inc., Cedar Grove, NJ, USA) was provided for use with the inhaled steroid only, as required by current guidelines [1-2]. At visit 2, randomized patients were requested to discontinue use of their own BDP pMDI, and BDP pMDI (Becotide $\mathbb{R} 250$ $\mu \mathrm{g} \cdot$ puff $^{-1}$, GlaxoWellcome) was provided to all. In addition, formoterol pMDI (Foradil $\mathbb{R}) 12 \mu \mathrm{g} \cdot$ puff $^{-1}$, Novartis, Switzerland) was provided to patients randomized thereafter.

Peak expiratory flow rate (PEF) (best of three measurements using a mini-Wright $(\mathrm{R})$ peak-flow meter), daytime symptoms $(0-4$ scale: $0=$ no symptoms - unrestricted activity; $4=$ symptoms at rest, routine activity affected, rescue medication does not control symptoms well), night time symptoms $(0-4$ scale: $0=$ did not wake-up because of breathing problems; $4=$ difficulty in sleeping due to breathing problems in spite of use of rescue medication) and use of rescue salbutamol were recorded daily in a diary by each participating patient (PEF and rescue salbutamol recorded both morning and evening).

All patients were asked to measure PEF at the same time in the morning and in the evening, and always prior to the administration of study medication. Any usage of salbutamol rescue within $6 \mathrm{~h}$ prior to a PEF measurement was also noted in the patient diary. Patients that made use of more than eight actuations per day of rescue salbutamol, for $>2$ consecutive days, were instructed to immediately contact their centre's investigator for assessment and initiation of appropriate therapy. The patient remained in the study only in the case of a short additional course of an oral corticosteroid. Addition of any other anti-asthmatic medication was considered as development of an exclusion criterion, and constituted sufficient reason for discontinuation of the particular patient from the study. However, patients that were discontinued from the study, for reasons as outlined above, were subsequently included in the exploratory analysis of the results. In addition, all adverse experiences were noted, by each investigator, in the appropriate section of the clinical record form (CRF). Serious adverse experiences were reported to the Regulatory Authorities within the required expedited time-lines. Finally, lung function was assessed spirometrically (forced expiratory volume in one second (FEV1) and forced vital capacity $(\mathrm{FVC})$ ) during clinic visits, following the ATS guidelines for spirometry [10].

\section{Patients}

A total of 159 patients, $\geq 18$ yrs old, were enrolled in the study. Patients were subsequently randomized to study treatment, if they fulfilled the following inclusion criteria: a symptom score (day and night) of two or greater on at least 4 of the 7 days during the second week of the run-in period, FEV1 before administration of an inhaled agonist $40-85 \%$ of the predicted normal for the patient [11], and a reversibility test with $200 \mu \mathrm{g}$ salbutamol demonstrating an increase in FEV1 of at least $15 \%$ from baseline value [1-2]. Finally, patients were required to have been using inhaled BDP aerosol for a least 1 month prior to enrolment, and at a constant daily dose of $500 \mu \mathrm{g}$.

Patients were excluded from participation for the following reasons; if they presented evidence of other clinically significant diseases, pregnant or lactating women, patients on $\beta$-blocker therapy or with hypersensitivity to sympathomimetic amines, those who were considered unable to comply with the study protocol and patients who had received a short course with an oral corticosteroid in the 6 weeks prior to enrolment, or more than three oral corticosteroid short courses during the year prior to enrolment.

\section{Statistical analysis}

Efficacy. The primary objective of this study was to compare the effect of the two treatment regimens on lung function. To achieve this, a confirmatory analysis was carried-out on a mean morning premedication PEF measured during the final 7 days of treatment (i.e. week 12 of treatment). Consequently for this analysis, only those patients who had completed the whole treatment period were included. Mean morning PEF was considered using analysis of covariance to estimate treatment contrasts. The baseline value calculated from the last seven days of the run-in period was fitted as a covariate. In addition to the estimate of the treatment difference, its 95\% confidence interval (CI) was calculated. An exploratory analysis was carried out on morning and evening PEF during the final 7 days before each monthly examination, on asthma scores, rescue medication used, premedication FEV1 measured at the clinic at 4, 8 and 12 weeks, and on the number of premature discontinuations. An analysis of covariance was performed to estimate the treatment contrasts and confidence intervals as above for the overall mean morning PEF, overall mean evening PEF, and the premedication FEV1 measured at the clinic. Asthma scores and rescue medication for the randomized treatment period were analysed using the test of VAN ELTEREN [12] stratified by centre using a mean score per patient for each variable. Finally, the number of premature discontinuations was considered descriptively.

The sample size was estimated in order to detect a difference of $25 \mathrm{~L} \cdot \mathrm{min}^{-1}$ using an estimated standard deviation of $48 \mathrm{~L} \cdot \mathrm{min}^{-1}$. Therefore, it was considered that $\sim 60$ evaluable patients per treatment group would give the study a power of $80 \%$ at the $5 \%$ significance level.

Safety. All patients randomized to treatment were included in the consideration of safety. This was assessed by the monitoring of adverse experiences. No formal statistical analysis was undertaken. Adverse experiences were summarized by World Health Organisation (WHO) class using two definitions as follows: 1) all adverse experiences; and 2) all adverse experiences considered by the investigator to be possible, probably or highly probably related to trial medication. 


\section{Results}

From a total of 159 patients initially enrolled in the study, 134 were randomized to treatments. Of these 134 patients, 69 were randomized to receive formoterol $12 \mu \mathrm{g}$ b.i.d. plus BDP $250 \mu \mathrm{g}$ b.i.d., and 65 were randomized to receive BDP $500 \mu \mathrm{g}$ b.i.d. (table 1). Accordingly, 25 patients were not randomized, as they did not present the required symptoms that would had qualified them for the set-up in the treatment that the study foresaw, and/or because they did not meet the strict reversibility "eligibility" criterion. Both groups were sufficiently matched as to demographic and baseline characteristics. Immediately after randomization, two patients did not return for scheduled examinations. Thus, 132 patients presented evaluable data. A total of 124 patients completed the trial, of which 122 presented with evaluable data for the confirmatory analysis (mean morning premedication PEF during the final 7 days of treatment). There were 10 premature discontinuations, four in the formoterol/BDP group and six in the higher BDP group. Two premature discontinuations (one in each group) were due to asthma deterioration that led to the development of an exclusion criterion (not allowed add-on therapy). One patient became pregnant during the course of the study and was discontinued. Five patients did not return for a scheduled visit (three on BDP and two on formoterol/BDP), including one patient in the higher BDP group who withdrew consent early in the study.

\section{Peak expiratory flow}

Morning premedication PEF increased in both the group of patients that received formoterol/BDP and the group of patients that received BDP alone, compared to baseline values. Confirmatory analysis carried out on the mean morning premedication PEF, measured during the final 7 days of the 12-week treatment period, demonstrated a treatment effect of $20.36 \mathrm{~L} \cdot \mathrm{min}^{-1}$ in the group of patients that received formoterol/BDP $(\mathrm{p}=0.021,95 \%$ CI 3.16237.560 ) over the group of patients that received BDP only (fig. 1).

Table 1. - Demographic data of the studied population

\begin{tabular}{lc}
\hline Age yrs & $43 \pm 14 / 9(18-72)$ \\
Sex M/F & $46 / 86$ \\
Weight kg & $71.8 \pm 13.2(35-100)$ \\
Height cm & $164 \pm 8.5(143-190)$ \\
PEF-morning* L·min & \\
FMR/BDP & $380.4 \pm 108.8$ \\
BDP & $356.4 \pm 96.2$ \\
PEF-evening* L·min & \\
FMR/BDP & $391.5 \pm 110.8$ \\
BDP & $361 \pm 96.6$ \\
FEV1* mL & \\
FMR/BDP & $2269 \pm 792$ \\
BDP & $2146 \pm 746$ \\
\hline
\end{tabular}

Data are mean \pm SD (range). M: male; F: female; PEF: peak expiratory flow; FMR/BDP: $12 \mu \mathrm{g}$ formoterol (FMR) b.i.d. 250 $\mu \mathrm{g}$ beclomethasone diproprionate (BDP) b.i.d. group; BDP: 500 $\mu \mathrm{g}$ BDP group; FEV1: forced expiratory volume in one second. *: The three lung function parameters were not significantly different between the two groups ( $p>0.05$ for each parameter).

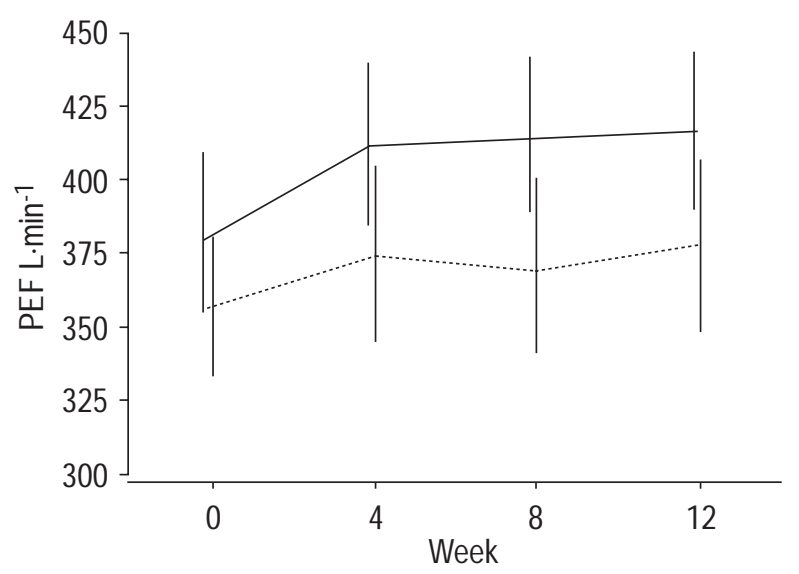

Fig. 1. - Mean morning pre-medication peak expiratory flow (PEF) during the final week before each monthly examination.
Formoterol $24 \mu \mathrm{g} \cdot \mathrm{day}^{-1}$ plus Beclomethasone dipropionate $500 \mu \mathrm{g} \cdot \mathrm{day}^{-1}$, - - - : Beclomethasone dipropionate $1,000 \mu \mathrm{g} \cdot \mathrm{day}^{-1}, 95 \%$ confidence intervals represented as solid vertical lines.

Mean morning premedication PEF during the week before the monthly clinic visits (weeks 4 and 8 ) was also higher in the formoterol/BDP group, and the difference was of statistical significance at week $8(\mathrm{p}=0.003)$. The overall mean morning premedication PEF for the entire treatment period was also significantly higher in the formoterol/BDP treatment group ( $\mathrm{p}=0.002$ ) (fig. 1). Evening premedication PEF also increased in both groups of patients compared to baseline. Mean evening pre-medication PEF during the week before the monthly clinic visits (weeks 4, 8, and 12) was numerically higher in the formoterol/BDP treatment group, compared to the higher BDP group, but it achieved statistical significance only during the week before visit 5 (week $8, \mathrm{p}<0.05$ ). The overall evening premedication PEF for the entire treatment period was found to be significantly higher in the formoterol/BDP group of patients as compared to the higher dose BDP group of patients $(\mathrm{p}<0.05)$ (figs. 2 and 3$)$.

\section{Asthma symptom scores}

Asthma symptom scores as recorded in the morning (for the previous night), and as recorded in the evening (for the

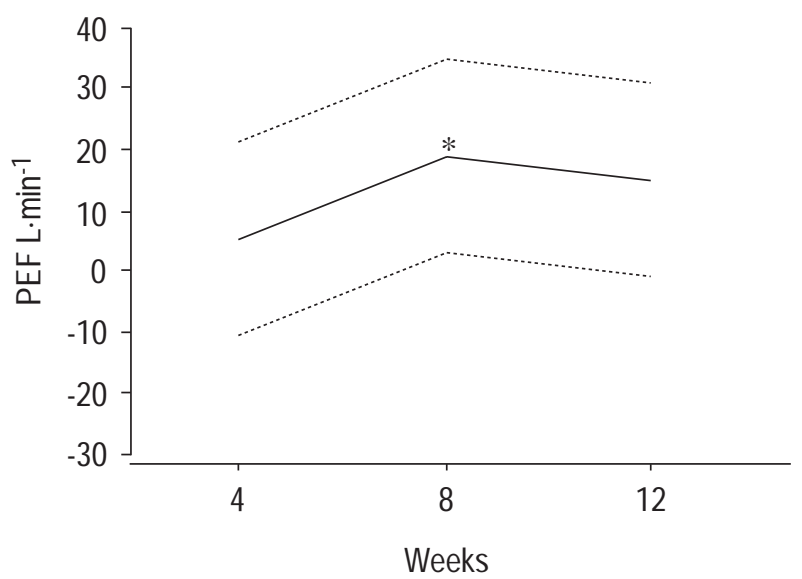

Fig. 2. - Formoterol and beclomethasone dipropionate treatment effect on evening pre-medication peak expiratory flow (PEF) (- $\longrightarrow$ ) and $95 \%$ confidence interval upper and lower limits (- - - ), *: p<0.05; overall $\mathrm{p}=0.044$. 


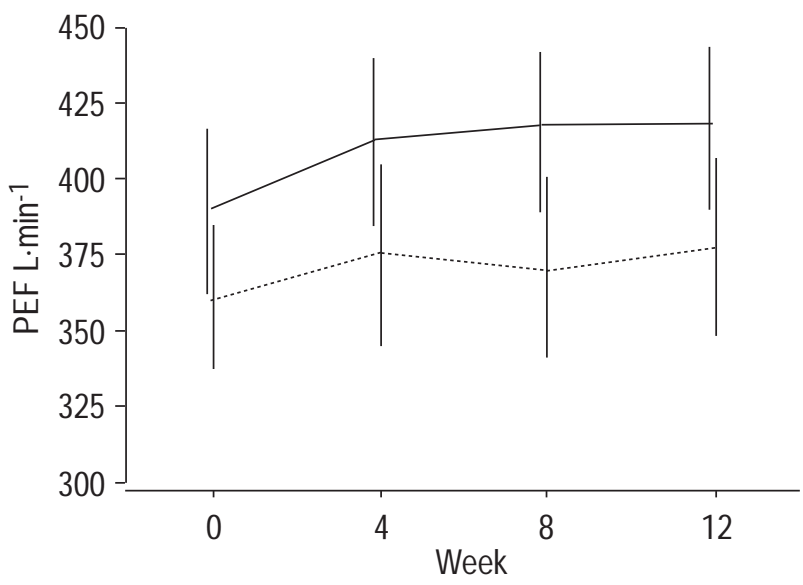

Fig. 3. - Mean evening pre-medication peak expiratory flow (PEF) during the final week before each monthly examination. - : Formoterol $24 \mu \mathrm{g} \cdot \mathrm{day}^{-1}$ plus Beclomethasone dipropionate $500 \mu \mathrm{g} \cdot \mathrm{day}^{-1}$, - - - : Beclomethasone dipropionate $1,000 \mu \mathrm{g} \cdot$ day $^{-1}, 95 \%$ confidence intervals represented as solid vertical lines.

previous day), decreased in both groups of patients compared to baseline. For the overall period, the difference between groups was again in favour of the group of patients that received formoterol plus BDP; day overall $\mathrm{p}=$ 0.001 , night overall $\mathrm{p}<0.001$ (fig. 4).

\section{Rescue medication}

The number of puffs of rescue medication (salbutamol) taken during the day and the evening/night, as recorded in the patients' diary, decreased in both groups of patients compared to baseline. For the overall period, the difference was again in favour of the group of patients that received formoterol plus BDP; day overall $\mathrm{p}<0.001$, night overall $\mathrm{p}=0.003$ (fig. 5 ).

\section{Spirometry}

Premedication FEV1, measured and recorded at each "monthly" clinic visit, also increased in both groups of patients compared to baseline, but the difference between them was statistically significant at week 8 . This was in favour of the administration of formoterol plus BDP $(\mathrm{p}<0.05)$ (fig. 6).

\section{Premature discontinuations}

There were a total of 10 premature discontinuations; four in the formoterol/BDP group and six in the higher dose BDP group. Two of them were caused by asthma deterioration (one in each treatment group). This deterioration led to additional treatment (e.g. theophylline), which in turn constituted an exclusion criterion (reason for discontinuation from the study).

\section{Adverse experiences}

Monitoring of adverse experiences (AEs) did not reveal differences between treatments. Both treatments were well tolerated. There were 74 AEs reported in the group of patients that received formoterol plus BDP, and 102 AEs in the group of patients that received higher dose BDP,
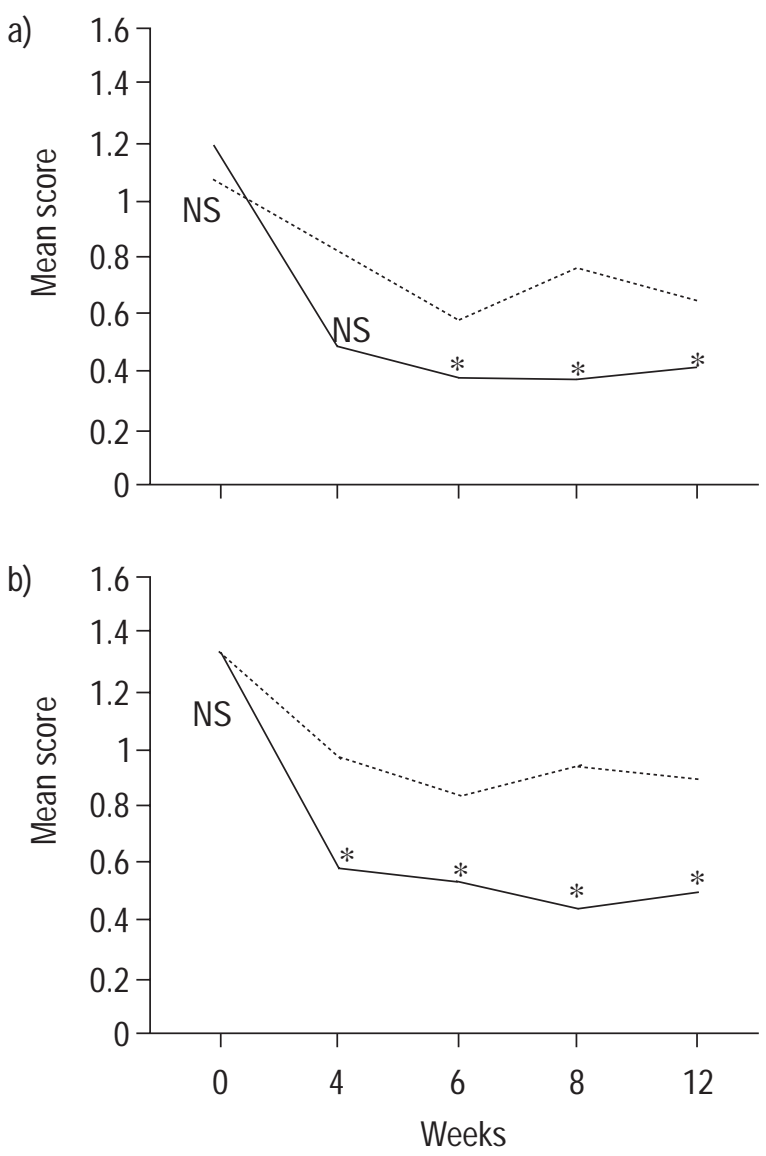

Fig. 4. - Mean asthma symptom score for the day (a) and the night (b) during the treatment period. — : Formoterol $24 \mu \mathrm{g} \cdot \mathrm{day}^{-1}$ plus Beclomethasone dipropionate $500 \mu \mathrm{g} \cdot \mathrm{day}^{-1}$, - . - : Beclomethasone dipropionate $1,000 \mu \mathrm{g} \cdot$ day $^{-1}$. $*$ : $\mathrm{p}<0.05$; NS: not significant; (a) overall $\mathrm{p}=0.001 ;$ (b) overall $\mathrm{p}<0.001$.

irrespective of causality (table 2). A total of seven AEs in the formoterol/BDP group and 27 in the higher BDP group were characterized by the reporting investigator as either possibly or probably related to trial medication. However, the majority of AEs were reported as mild in severity. Furthermore, only two patients presented with a serious AE (one in each group of patients). The patient from the group of patients receiving formoterol plus BDP, was hospitalized for sinusitis. The other patient of the group of patients that received the higher dose BDP, required a brief hospitalization for asthma deterioration and was subsequently discontinued from the trial due to the addition of other anti-asthma therapy (not allowed by the protocol).

\section{Short courses of oral steroids}

A total of 11 patients received treatment with oral corticosteroids during the study. Eight patients belonged to the group that was under treatment with formoterol/BDP, and three in the group that was under treatment with the higher BDP dose. It should be noted that two patients in the formoterol/BDP group and two patients in the higher BDP group were identified, after study completion, as protocol violators. All four had required, and received, a short course with an oral corticosteroid during the run-in period 

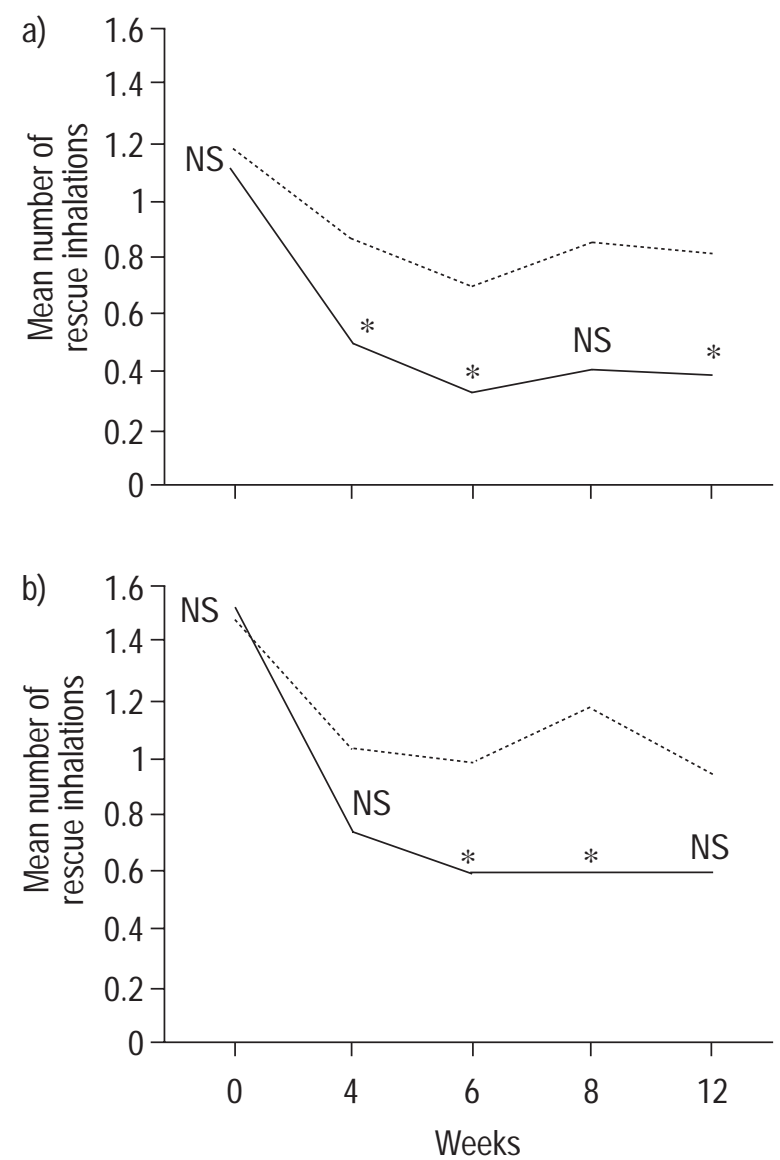

Fig. 5. - Mean number of rescue salbutamol inhalations taken during the day (a) and during the night (b) during the treatment period. Formoterol $24 \mu \mathrm{g} \cdot \mathrm{day}^{-1}$ plus Beclomethasone dipropionate $500 \mu \mathrm{g} \cdot \mathrm{day}^{-1}$, - - - : : Beclomethasone dipropionate $1,000 \mu \mathrm{g} \cdot$ day $^{-1}$. *: $\mathrm{p}<0.05$; ns: not significant; (a) overall $\mathrm{p}<0.001$; (b) overall $\mathrm{p}=0.003$.

of the study. According to the protocol, this was indicative of unstable disease, and constituted an exclusion criterion. Assessed using the Chi-square test, these differences ( 8 versus $3 ; 6$ versus 1 ) have significance values of $\mathrm{p}=0.14$ and $\mathrm{p}=0.06$, respectively.

\section{Discussion}

These results show that in adult asthmatic patients still presenting with symptoms, the addition of formoterol fumarate solution aerosol $12 \mu \mathrm{g}$ twice daily to an existing daily dose of $500 \mu \mathrm{g}$ of inhaled BDP resulted in a greater improvement in lung function and better control of symptoms, when compared to an increase of the inhaled steroid daily dose to $1,000 \mu \mathrm{g} \cdot \mathrm{day}^{-1}$. The safety and tolerability of both regimens did not differ, and was judged as satisfactory.

These results are in agreement with the results from previous work with salmeterol. Both GREENING et al. [7] and WoOLCOCK et al. [8] reported improved lung function and better control of symptoms with the regular addition of salmeterol than with an increase in daily doses of BDP from $400-1,000 \mu \mathrm{g}$ and from $1,000-2,000 \mu \mathrm{g}$, respectively.

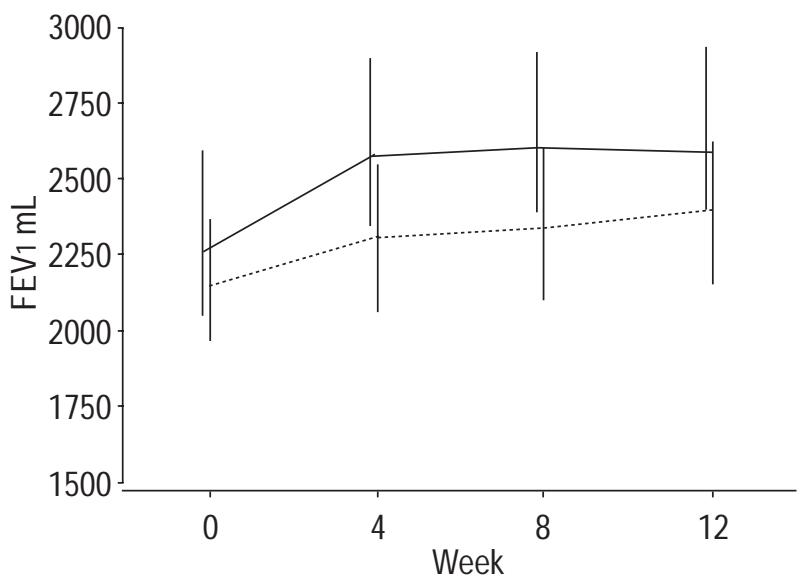

Fig. 6. - Mean forced expiratory volume in one second (FEV1) at the monthly examinations at the clinic. $\longrightarrow$ : Formoterol $24 \mu \mathrm{g} \cdot$ day $^{-1}$ plus Beclomethasone dipropionate $500 \mu \mathrm{g} \cdot \mathrm{day}^{-1},----$ : Beclomethasone dipropionate $1,000 \mu \mathrm{g} \cdot$ day $^{-1}$. $95 \%$ confidence intervals represented as solid vertical lines.

Formoterol, like salmeterol, is a long-acting $\beta_{2}$-agonist developed for use as a maintenance treatment in asthma $[13,14]$. Thus, it is prescribed to be taken twice daily, in order to prevent symptoms, rather on an "as needed basis" in order to treat symptoms. Questions to be answered over its regular use are the same ones that maintain the controversy over the regular use of short-acting $\beta_{2}$ agonists [4-6]. That is, does regular use of formoterol improve overall control of asthma? To this the asthma exacerbation rate is considered the most important indicator [15]. In the present study, there were only two observed exacerbations that necessitated additional addon therapy that was not allowed by the protocol (i.e. xanthine derivatives, inhaled anticholinergics, inhaled $\beta_{2}$ agonists other than the study medication and cromones), one in each group, and none met the definition of serious (hospitalization, life-threatening or death). In addition, day and night asthma symptom scores and use of rescue salbutamol, were significantly less in the formoterol/BDP group compared to the higher dose BDP group.

However, it should be noted that 11 patients (eight patients in the combination group and three in the higher dose BDP group, $\mathrm{p}=0$. 14) in this study required "short course" oral steroid intake. If the protocol violators were excluded, seven patients (six in the combination group and one in the higher dose BDP group, $\mathrm{p}=0-06$ ) required "short course" oral steroid intake. While these differences did not reach traditional levels of statistical significance, they are

Table 2. - Number and percentage of adverse experiences (AEs) between treatments

\begin{tabular}{lrrrrr}
\hline \multirow{2}{*}{$\begin{array}{l}\text { Trial treatment } \\
\text { Causality }\end{array}$} & \multicolumn{2}{c}{ Formoterol/BDP } & & \multicolumn{2}{c}{ BDP } \\
\cline { 2 - 3 } \cline { 5 - 6 } \cline { 5 - 6 } & AEs & \multicolumn{1}{c}{$\%$} & & AEs & $\%$ \\
\hline Not related & $54 / 74$ & 72.79 & & $59 / 102$ & 57.84 \\
Unlikely & $13 / 74$ & 17.57 & & $16 / 102$ & 15.69 \\
Possible & $5 / 74$ & 6.76 & & $24 / 102$ & 23.53 \\
Probable & $1 / 74$ & 1.35 & & $1 / 102$ & 0.98 \\
Highly probably & $1 / 74$ & 1.35 & & $2 / 102$ & 1.96 \\
Total & 74 & 100 & & 102 & 100 \\
\hline
\end{tabular}

BDP: beclomethasone dipropionate. 
similar to the findings of PAuwels et al. [9], who showed that whereas the addition of formoterol to low dose of budesonide was a more effective regimen than increasing the dose of budesonide in terms of lung function, symptoms and $\beta$-agonist use, is less effective in terms of reducing exacerbations requiring oral steroid use and/or presenting with a significant decrease in PEF.

It has also been suggested in the past that this improved control of symptoms may lead to a "masking" of the underlying decreased asthma control, and this may develop a tendency for more severe exacerbations in subsets of patients [15]. Pauwels et al. [9] found that the rates of severe and mild exacerbations were reduced by 26 and $40 \%$ when formoterol was added to $200 \mu \mathrm{g} \cdot \mathrm{day}^{-1}$ and 800 $\mu \mathrm{g} \cdot \mathrm{day}^{-1}$ budesonide, respectively. It is believed that these observations are reassuring when the current findings in relation to exacerbations requiring oral corticosteroids are considered.

Another vital question would be whether regular use of formoterol influences lung function or leads to a rebound increase in bronchial responsiveness on discontinuation of therapy and a reduction in bronchodilator effect. The authors' experience from this study is consistent with previous studies with formoterol that have shown no reduction in lung function, worsening in bronchial responsiveness or reduction in bronchodilator effect [16-19].

Overall, the current investigation into the efficacy and safety of treatment in this 3-month study failed to identify an area of concern with regard to decreased asthma control, and resulting hazard to the patient. However, further investigations should be undertaken. These should be of longer duration, should include different doses of inhaled corticosteroids (both higher and lower) and large numbers of patients. As this study along with the findings of PAuweLs et al. [9] tend to indicate that increasing the maintenance dose of inhaled corticosteroids might be a more appropriate initial therapeutic step in the "subset" of patients with repeated severe exacerbations, such future studies should present with sufficient statistical power that will allow for definite conclusions in this vital area of concern.

In conclusion, the addition of formoterol fumarate should be considered as an alternative to increasing the inhaled corticosteroid dose in the inadequately controlled asthmatic.

\begin{abstract}
Acknowledgements. The authors would like to thank the following physicians/research scientists for enrolling patients at their respective centres, and/or their overall important contributions to the successful completion of this study: S. Tzannes, M. Demadi, G. Mathioudakis, A. Chaniotou, G. Blazoyiannakis, C. Trabari and A. Tsakatikas (Athens); P. Christaki, Y. Pegios and E. Vlachoyianni (Thessaloniki); K. Stachouli (Ioannina); N. Charokopos and K. Spyropoulos (Patra); K. Kallergis and N. Tzanakis (Crete); and D. Till (Horsham, UK).
\end{abstract}

\section{References}

1. National Institutes of Health. National Heart, Lung, and Blood Institute. International Consensus Report on Diagnosis and Treatment of Asthma. Eur Respir J 1992; 5: 601-641.
2. National Institutes of Health. National Heart, Lung, and Blood Institute. Global Initiative for Asthma: Global Strategy for Asthma Management and Prevention NHLBI/ WHO Workshop Report March 1993. NIH Publication 1995, No. 95-3659.

3. Pauwels R. The clinical use of $\beta$-receptor agonists, for and against. Life Sciences 1993; 52: 2171-2179.

4. Sears MR, Taylor DR, Print CG, et al. Regular inhaled beta-agonist treatment in bronchial asthma. Lancet 1990; 336: 1391-1396.

5. Spitzer WO, Suisa S, Ernst P, et al. The use of b-agonists and the risk of death and near death from asthma. $N$ Engl $J$ Med 1992; 326: 501-506.

6. Mullen M, Mullen B, Carey M. The association between b-agonists use and death from asthma. A meta-analytic integration of case-control studies. JAMA 1993; 270: $1842-1845$.

7. Greening AP, Ind PW, Northfield M, Shaw G. Added salmeterol versus higher-dose corticosteroid in asthma patients with symptoms on existing inhaled corticosteroid. Lancet 1994; 344: 219-224.

8. Woolcock A, Lunback B, Ringdal N, Jacques LA. Comparison of addition of salmeterol to inhaled steroids with doubling of the dose of the inhaled steroids. Am J Respir Crit Care Med 1996; 153: 1481-1488.

9. Pauwels RA, Loefdahl C-G, Postma DS, et al. Effect of inhaled formoterol and budesonide on exacerbations of asthma. N EngI J Med 1997; 337: 1405-1411.

10. American Thoracic Society. Standardization of spirometry, 1994 update. Am J Respir Crit Care Med 1995; 152: $1107-1136$.

11. Quanjer PH, Tammeling GJ, Cotes JE, Pedersen OF, Peslin R, Yernault JC. Lung volumes and forced ventilatory flows: report Working Party Standarization of Lung Function Tests. European Community for Steel and Coal: official statement of the European Respiratory Society. Eur Respir J 1993; 6 (SuppI 16): 5-40.

12. van Elteren PH. On the combination of independent twosample tests of Wilcoxon. Bulletin de l'Institute International de Statistique 1960; 37: 351-361.

13. Anderson GP. Formoterol: Pharmacology, molecular basis of agonism, and mechanism of long duration of a highly potent and selective $\beta$-adrenoreceptor agonist bronchodilator. Life Sciences 1993; 152: 2145-2160.

14. Tattersfield AE. Long-acting $\beta_{2}$-agonists. Clin Exper Aller 1992; 22: 600-605.

15. Devoy MAB, Fuller RW, Palmer JBD. Are there any detrimental effects of the use of inhaled long-acting $\beta_{2}$-agonists in the treatment of asthma? Chest 1995; 107: 1116-1124.

16. Kesten S, Chapman KR, Broder I, et al. Sustained improvement in asthma with long term use of formoterol fumarate. Annals Allergy 1992; 69: 415-420.

17. Kesten S, Chapman KR, Broder I, et al. A three-month comparison of twice daily inhaled formoterol versus four times daily inhaled albuterol in the management of stable asthma. Am Rev Respir Dis 1991; 144: 622-625.

18. Yates DH, Sussman HS, Shaw MJ, Barnes PJ, Chung KF. Regular formoterol treatment in mild asthma: effect on bronchial responsiveness during and after treatment. $\mathrm{Am} \mathrm{J}$ Respir Crit Care Med 1995; 152: 1170-1174.

19. Arvidsson P, Larsson S, Loefdahl C-G, Melander B, Svedmyr N, Wahlander L. Inhaled formoterol during one year in asthma: a comparison with salbutamol. Eur Respir $J$ 1991; 4: 1168-1173. 